\title{
Challenges in Managing Hospitalized HIV Infected Persons with Low Absolute CD4 and Preserved CD4 Percentage
}

\author{
Steven M. Bobula, Carl J. Fichtenbaum \\ Division of Infectious Diseases, Department of Internal Medicine, University of Cincinnati College of Medicine, Cincinnati, USA. \\ Email: carl.fichtenbaum@uc.edu
}

Received May $18^{\text {th }}, 2012$; revised June $21^{\text {st }}, 2012$; accepted July $8^{\text {th }}, 2012$

\begin{abstract}
Background: HIV infected persons are at risk for opportunistic illnesses based upon severity of immune deficiency. Management is generally based upon the most recent absolute CD4 count. We hypothesized there is a group of patients with a low absolute CD4 count and preserved CD4 percentage that are at low risk of AIDS-related opportunistic illnesses (OI). Methods: A retrospective review of medical records in HIV-infected persons hospitalized from 2004-2006. Individuals without CD4 counts available within 180 days of admission and during hospitalization were excluded. Patients with a decrease in the absolute CD4 count during hospitalization and stable CD4 percentage were compared to the rest of the cohort. Appropriate management was defined using DHHS guidelines for the prevention and treatment of opportunistic illnesses in HIV infection. Results: 464 patients had 978 hospitalizations. In 221 hospitalizations $(\mathrm{N}=$ 161 patients) inpatient and outpatient CD4 counts were available. In 35 hospitalizations ( $\mathrm{N}=25$ patients) the absolute CD4 count declined with stable CD4 percent (cases). Cases had an average decline in CD4 of $-197 \mathrm{cells} / \mathrm{mm}^{3}$ compared to -5 cells $/ \mathrm{mm}^{3}$ in the comparator group. $30 \%$ of comparators had AIDS defining OI's compared to none in the case group $(\mathrm{p}=0.01)$. Management outside of DHHS guidelines was more common in cases compared to the comparator group ( $49 \%$ vs $30 \%, p=0.048)$. The median length of stay was prolonged in cases with management outside guidelines compared to appropriately managed persons in the comparator group ( 7 days vs 3.5 days, $p=0.03)$. Conclusion: In persons on potent antiretroviral therapy, abrupt declines in absolute CD4 counts without an accompanying change in CD4 percentage are associated with a low risk of AIDS related opportunistic infection, a higher rate of in-patient management outside DHHS guidelines, and a more prolonged length of stay.
\end{abstract}

Keywords: HIV; CD4 Count; Risk Stratification; Acute Illness; Opportunistic Infections

\section{Introduction}

T-lymphocytes have been used to assess risk for the development of Acquired Immune Deficiency Syndrome (AIDS) related opportunistic illnesses (OIs) for 3 decades $[1,2]$. The risk for developing specific infections is typically defined by the absolute number of circulating CD4 positive lymphocytes [3]. Although risk stratification may be estimated using the percentage of circulating CD4 lymphocytes, this is uncommon in clinical practice. Most clinicians utilize the most recent absolute CD4 count to estimate the severity of HIV associated immune deficiency.

In persons hospitalized with HIV, recent CD4 counts and HIV RNA levels may not always be available. Consequently, individuals hospitalized with HIV often have CD4 counts and HIV RNA levels obtained within the first day or two to risk stratify the likelihood of various
OIs.

We hypothesized that some HIV infected adults hospitalized for acute conditions and illnesses are incorrectly risk stratified and managed based upon acutely measured lower absolute CD4 counts. This decline in the number of circulating lymphocytes occurs during stress reactions associated with acute disease states. The accompanying CD4 percentage is likely to be preserved at near prehospitalization levels enabling the clinician to distinguish between those with and without a biologically important decline. These patients are characterized by ongoing use of antiretroviral therapy with prior HIV viral loads near the limit of detection and good self-reported recent adherence to treatment. Therefore, we evaluated the impact of measuring inpatient CD4 counts on influencing the diagnostic approach, empiric management, outcome and length of stay for HIV infected persons who were hospitalized. 


\section{Methods}

\subsection{Setting}

An academic, 700-bed, urban, public hospital located in Cincinnati, Ohio. The study was approved by the institutional review board at the University of Cincinnati.

\subsection{Population and Study Design}

A retrospective review of the medical records of all HIV-infected persons admitted to University Hospital from 2004-2006. Participants were excluded from further analysis if they were less than eighteen years of age, did not have HIV infection documented by positive ELISA and confirmed by Western Blot, were not being prescribed antiretroviral therapy for at least 4 months, or did not have CD4 counts drawn during their inpatient stay and within 180 days prior to admission.

Patients were divided into case and comparator groups based the stability or instability of their inpatient and outpatient CD4 counts and percentages. Cases were arbitrarily classified if there was a $>20 \%$ decline in absolute
CD4 count with a $<20 \%$ decline in absolute CD4 percent compared to prior outpatient values obtained within 180 days of hospitalization. For example, a patient hospitalized where the outpatient and inpatient absolute CD4 counts and percentages were $372 / 19 \%$ and $180 / 18 \%$, respectively. We chose this level of change based upon a priori clinical rationale that there is significant variability in the CD4 assays and that a minimum of at least $20 \%$ change was likely to be biologically more important. Patients not meeting this definition were used as a comparator group (Figure 1).

\subsection{Data Collection}

Using standardized data collection forms, the medical records were abstracted for demographic data, admission and discharge diagnoses; specialty of health care providers involved in the patient's care; use of antiretroviral therapy; length of hospitalization; antibiotic utilization, use of OI prophylaxis and treatment; use of adjunctive corticosteroids; procedures performed; microbiologic studies; laboratory tests; complications from medications

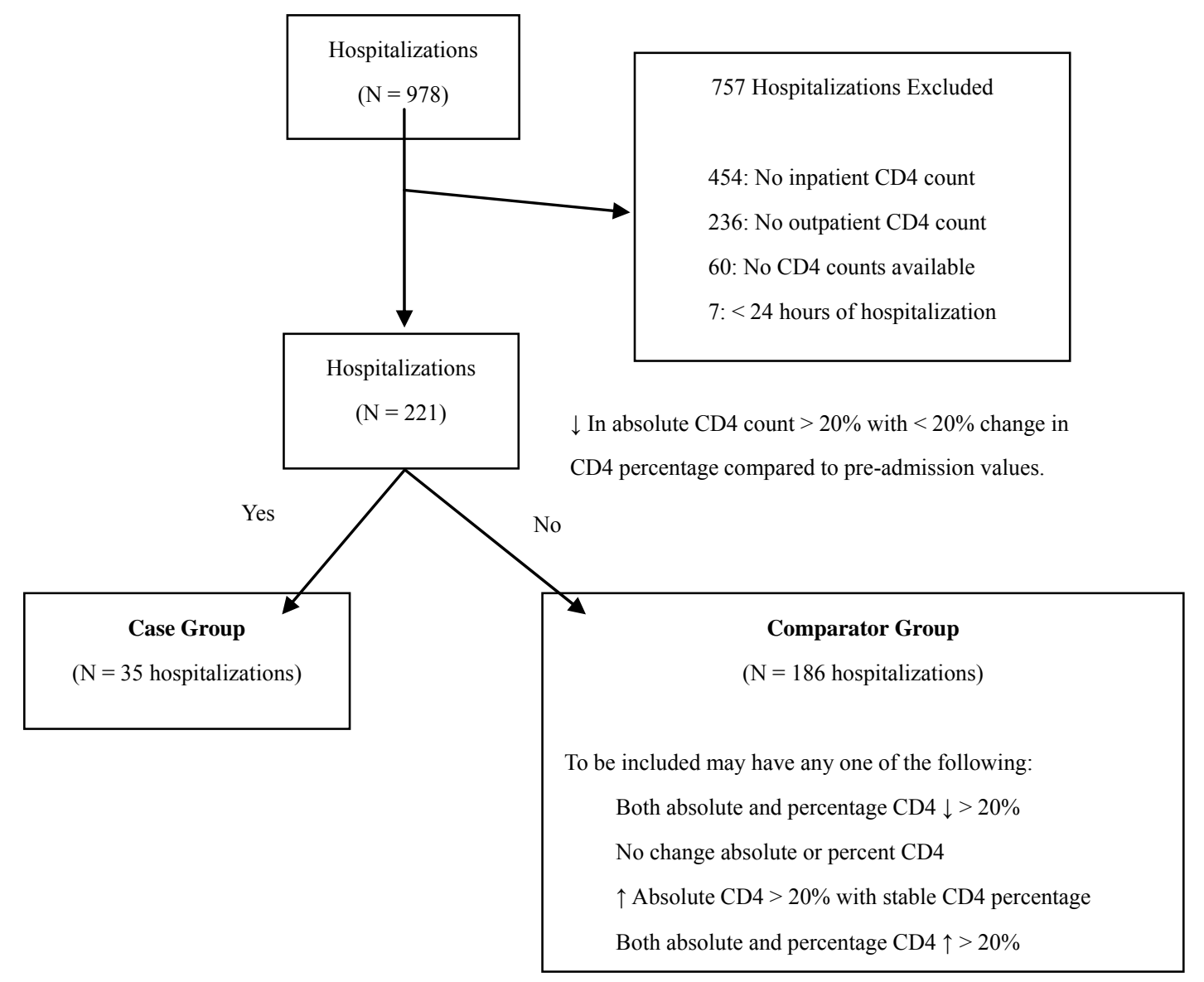

Figure 1. Consort diagram of study population. A description of the study population evaluated over 3 years of the study. The case group was defined by those admitted with a $>20 \%$ decline in absolute CD4 count without a significant change in CD4 percentage. The comparator group was defined by those with either no change in CD4 or a significant change $>20 \%$ in both absolute and percentage of CD4 cells. 
or procedures; and hospital outcomes. All charts were reviewed by both authors. Management within or outside standard guidelines were assigned regardless of whether the subject was later classified as a case or comparator. When there was disagreement between the authors, a consensus method was used to resolve differences.

\subsection{Definitions}

Appropriateness of management was determined by comparing the patients' management relative to current Department of Health and Human Services (DHHS) and other available guidelines on the treatment and prevention of OIs in persons infected with HIV [3-6]. Risk stratification to determine appropriateness of care was based upon the most recent outpatient CD4 count. If there was more than one measurement within 180 days, we used the one most proximate to hospitalization. We assumed that if there was no documented interruption in antiretroviral therapy, absolute CD4 counts would be unlikely to decline significantly in the absence of intercurrent illness or laboratory error. An HIV specialist was defined as a practitioner who routinely provides inpatient and outpatient care to individuals infected with HIV.

\subsection{Laboratory Methods}

HIV plasma RNA levels (HIV viral load) were measured using the Amplicor HIV-1 monitor test, version 1.5, in the local affiliated laboratory. Lymphocyte subset quantification, including CD4 counts, was obtained by flow cytometry.

\subsection{Statistical Analysis}

The primary objective was to compare differences in adherence to DHHS management guidelines between the case and comparator groups. Secondary objectives included use of unneeded prophylaxis and treatment for $P$. jirovecii pneumonia and disseminated M. avium complex; determining if the involvement of an HIV specialist in the subject's care led to a lower rate of deviation of management from standard guidelines; determining if there were differences in the occurrence of AIDS defining opportunistic infections; and evaluating the effect of adherence to management guidelines on length of stay, morbidity, and mortality; however, the study was not powered to determine a difference in mortality.

The study was designed to prove an absolute $10 \%$ difference in deviation from management guidelines in persons with an acute decline in absolute CD4 counts obtained during their hospitalization. We estimated a sample size of 120 hospitalizations was needed, assuming that the rate of management deviation in the comparator population is approximately $19 \%$ (Power of $80 \%$, $P<0.05$, two tailed). A $P$ that was less than or equal to
0.05 was considered statistically significant.

All data was analyzed by using SAS 9.1.3 (SAS Institute, Cary, North Carolina). Chi squared testing was performed to compare categorical variables. Nonparametric methods were used to compare continuous variables. Categorical variables are reported with number and percentage of total population. Median results with reported minimum and maximum ranges were used to report most continuous variables. For analysis of HIV viral loads, we assigned arbitrary next value to those below the limit of detection. For example, those with an HIV viral load < 50 copies $/ \mathrm{mL}$ were assigned a value of 49 copies $/ \mathrm{mL}$ for statistical analysis.

\section{Results}

There were 978 hospitalizations of 464 unique patients from 2004-2006. 221 hospitalizations had inpatient and outpatient CD4 counts available. Of these, 35 hospitalizations (25 patients) had a decreased absolute CD4 count $(>20 \%)$ on admission without a significant decline in CD4 percentage $(<20 \%)$. The remaining 186 hospitalizations (136 patients) comprised the comparator group. This included persons where the absolute CD4 count remained stable or both the absolute CD4 and percentage both declined or, increased. There were no statistically significant differences between the groups with respect to age, gender or race (Table 1). Comorbid conditions present at the time of hospitalization were not significantly different between the two groups. An HIV expert was involved in the management of $49 \%$ of case hospitalizations and $45 \%$ of comparator hospitalizations $(p=0.71)$.

Three deaths occurred during the study, two in the comparator group and one in the case group. In the comparator group, a 47-year-old man died with disseminated

Table 1. Study population demographics.

\begin{tabular}{ccc}
\hline Case Group & $\begin{array}{c}\text { (N=25) } \\
\text { (Range) } \\
\text { Gender }\end{array}$ & $\begin{array}{c}\text { Comparator } \\
\text { Group }\end{array}$ \\
Male & $44(27,65)$ & $42(21,65)$ \\
Fedian Age in Years & $80 \%$ & $80 \%$ \\
Race & $20 \%$ & $20 \%$ \\
African American & & \\
Caucasian & $48 \%$ & $64 \%$ \\
Hispanic & $48 \%$ & $32 \%$ \\
Other/Not Identified & $4 \%$ & $2 \%$ \\
\hline
\end{tabular}

*Represents number of patients not hospitalizations. 
T. gondii and M. avium disease. His outpatient and inpatient absolute CD 4 counts and percentages were $<1$ cells per cu $\mathrm{mm} / 0 \%$ and 9 cells per cu $\mathrm{mm} / 7 \%$, respectively, and had an HIV viral load that was greater than 100,000 copies $/ \mathrm{mL}$ despite self-reported adherence with highly active antiretroviral therapy (HAART). The second comparator group death involved a 39-year-old woman who died from $P$. jirovecii pneumonia. Her outpatient absolute CD4 count and percentage of 179 cells per cu $\mathrm{mm} / 17 \%$ had decreased to 26 cells per cu $\mathrm{mm} / 8 \%$ on admission. She had been intermittently adherent with HAART and had an HIV viral load greater than 750,000 copies $/ \mathrm{mL}$. The case group death involved a 49 -year-old man with bacterial meningitis who presented with an inpatient absolute CD4 count and percentage of 43 cells per cu $\mathrm{mm} / 13 \%$ (outpatient values of 242 cells per cu $\mathrm{mm} / 16 \%)$. He had been reportedly adherent with
HAART and had a HIV viral load of 16,800 copies $/ \mathrm{mL}$. The only minor deviation from standard management observed in these three patients involved failure to administer prophylaxis for M. avium complex in the second comparator case.

Patients in the case group had higher CD4 counts and percentages before ( 407 cells per cu $\mathrm{mm} / 20 \%$ versus 101 cells per cu $\mathrm{mm} / 18 \%, P<0.0001)$, during (180 cells per cu $\mathrm{mm} / 18 \%$ versus 101 cells per cu $\mathrm{mm} / 10 \%$ with $P<$ 0.05 ), and after hospital admission (373 cells per cu $\mathrm{mm} / 19 \%$ versus 159 cells per cu $\mathrm{mm} / 10 \%$ with $P=0.001$ ) than the comparator group. The case group was also noted to have lower median pre-admission viral loads than the comparator group (476 copies $/ \mathrm{mL}$ compared to 31,724 copies $/ \mathrm{mL}, P=0.001$ ). The acute decline in CD4 count seen in the case group generally reversed when subjects were retested as outpatients (Table 2) after discharge.

Table 2. CD4 Counts and HIV-1 RNA Levels.

\begin{tabular}{|c|c|c|c|}
\hline & Case Group $(\mathrm{N}=35)$ & $\begin{array}{l}\text { Comparator Group } \\
(\mathrm{N}=186)\end{array}$ & $P$-value \\
\hline \multicolumn{4}{|c|}{ Absolute Median CD4 count in cell $/ \mathrm{mm}^{3}$ (ranges) } \\
\hline Values between $6-12$ months before admission & $343(39,953)$ & $138(0,920)$ & 0.0002 \\
\hline Values within 6 months before admission & $407(118,1,252)$ & $101(0,1,531)$ & $<0.0001$ \\
\hline Values during admission & $180(22,777)$ & $101(0,1738)$ & 0.05 \\
\hline Change in absolute CD4 from pre-admission ${ }^{\mathrm{a}}$ & $-197(-484,-68)$ & $-5(-575,780)$ & $<0.0001$ \\
\hline Absolute CD4 1 - 3 months after admission & $333(71,1,128)$ & $159(0,1,344)$ & 0.001 \\
\hline Change in absolute CD4 after discharge ${ }^{\mathrm{b}}$ & $149(-294,680)$ & $24(-202,687)$ & $<0.0001$ \\
\hline \multicolumn{4}{|l|}{ Median CD4 percentage (ranges) } \\
\hline Values between 6 - 12 months before admission & $20(7,38)$ & $10(0,50)$ & $<0.0001$ \\
\hline Values within 6 months before admission & $20(11,44)$ & $8(0,50)$ & $<0.0001$ \\
\hline Values during admission & $18(11,40)$ & $10(0,38)$ & $<0.0001$ \\
\hline Change in percent $\mathrm{CD} 4$ from pre-admission ${ }^{\mathrm{a}}$ & $-3(-14,8)$ & $0(-18,19)$ & $<0.0001$ \\
\hline Percent CD4 1 - 3 months after admission & $19(7,43)$ & $10(0,50)$ & 0.0004 \\
\hline Change in percent CD4 after discharge ${ }^{\mathrm{b}}$ & $2(-9,15)$ & $0(-10,37)$ & 0.07 \\
\hline \multicolumn{4}{|c|}{ Median HIV-1 RNA in copies/mL (ranges) } \\
\hline Values between 6-12 months before admission & $5580(<50,>750,000)$ & $20,400(<50,>750,000)$ & 0.51 \\
\hline Values within 6 months before admission & $476(<50,269,000)$ & $31,724(<50,>750,000)$ & 0.001 \\
\hline Values during admission & $399(<50,365,000)$ & $26,550(<50,>750,000)$ & 0.17 \\
\hline Values 1 - 3 months after discharge & $745(<50,>750,000)$ & $2311(<50,>750,000)$ & 0.40 \\
\hline
\end{tabular}

${ }^{\mathrm{a}}$ Difference between values measured within 6 months of admission compared to those measured during admission. ${ }^{\mathrm{b}}$ Difference between values measured during admission compared to those measured 1 - 3 months after discharge. Note: All ranges represent minimum and maximum values. 
Patients that presented with a sudden decline in absolute CD4 were more often diagnosed with febrile illnesses as opposed to non-febrile illnesses (Table 3). Febrile illnesses were noted in $71 \%$ of case group patients compared to $49 \%$ of those in the comparator group $(P=$ 0.01). Lobar pneumonias were the most common febrile illness diagnosed in both groups, and were found more frequently in cases than comparators $(P=0.0007)$. AIDS defining OIs were common $(30 \%)$ in the comparator group but were not observed in the case group $(P=0.01)$. There was a non-significant trend of more episodes of bacteremia, sepsis, or urinary tract infections in the case group $(P=0.09)$. Diagnoses in the remaining $29 \%$ of the case group without febrile illness included multiple organ system trauma, craniofacial injuries, and acute long bone fractures. Case and comparator hospitalizations were remarkably similar regarding severity of illness. Intensive care unit management was required $20 \%$ of cases and $11 \%$ of comparators $(P=0.13)$. Both groups had median APACHE-II scores of $8(P=0.88)$.

Management outside standard guidelines was observed in $49 \%$ of patients hospitalized with an abrupt decline in absolute CD4 count without a significant change in CD4 percent (Case group). In contrast, $30 \%$ of patients in the comparator group were managed outside standard guidelines $(P=0.048)$. HIV experts were involved in $41 \%$ of case hospitalizations and $43 \%$ of comparator hospitalizations that were not managed per guidelines $(P=0.86)$. The most common reasons for deviation from management by guidelines were generally minor and included administration of prophylaxis for $P$. jirovecii and $M$. avium when they would not have been indicated. In several instances management deviations included use of laboratory studies and procedures (bronchoscopy or lumbar puncture) that were not absolutely required (Table 4). An example of a more significant deviation from standard guideline management involved a 64-year-old man with outpatient and inpatient absolute CD4 count and CD4 percentage of 472 cells per cu $\mathrm{mm} / 16 \%$ and 157 cells per cu mm/13\%, respectively, admitted for pneumonia (Case group). His HIV viral load prior to admission was $<50$ copies $/ \mathrm{mL}$. A lobar infiltrate was noted on the chest radiograph. According to the physician notes, the bronchoscopy was ordered to evaluate for $P$. jirovecii specifically due to the absolute CD4 count being less than 200 cells per cu mm. In this case, bacterial pneumonia was retrospectively judged to be far more likely. The absolute CD4 was $<200$ cells/cu mm because of the relative lymphopenia due to a "left shift" with many neutrophils circulating in a patient with a presumed bacterial pneumonia. Another example of deviation from standard management occurred in a 48-year-old male with outpatient and inpatient absolute CD4 count and CD4 percentage of $958 / 30 \%$ and $1738 / 29 \%$, respectively that was admitted for evaluation of seizure (Comparator group). The patient did not have fever and outpatient HIV viral load was $<50$ copies $/ \mathrm{mL}$. Review of the patient's chart noted that the primary team responsible for his care noted that he was HIV infected and specifically performed lumbar puncture to assess for a possible AIDS-defining OI.

Table 3. Diagnoses during hospitalizations.

\begin{tabular}{|c|c|c|c|}
\hline & Case Group ( $\mathrm{N}=35)$ & Comparator Group $(\mathrm{N}=186)$ & $P$-value \\
\hline Non-febrile illnesses & $29 \%$ & $51 \%$ & 0.01 \\
\hline Febrile Illnesses $^{\mathrm{a}}$ & $71 \%$ & $49 \%$ & 0.01 \\
\hline Pulmonary ${ }^{\mathrm{b}}$ & $46 \%$ & $19 \%$ & 0.0007 \\
\hline Bacteremia/Sepsis & $17 \%$ & $8 \%$ & 0.09 \\
\hline Urinary Tract Infection & $9 \%$ & $3 \%$ & 0.09 \\
\hline AIDS Defining $\mathrm{OI}^{\mathrm{c}}$ & $0 \%$ & $16 \%$ & 0.01 \\
\hline Skin and Soft Tissue ${ }^{\mathrm{d}}$ & $3 \%$ & $5 \%$ & 0.53 \\
\hline Gastrointestinal $^{\mathrm{e}}$ & $3 \%$ & $2 \%$ & 0.61 \\
\hline Immune Reconstitution Syndrome & $0 \%$ & $2 \%$ & 0.38 \\
\hline Viral Syndrome & $0 \%$ & $1 \%$ & 0.54 \\
\hline Fever of Unknown Origin & $3 \%$ & $3 \%$ & 0.96 \\
\hline Other Miscellaneous Illnesses & $9 \%$ & $1 \%$ & 0.01 \\
\hline
\end{tabular}

${ }^{\mathrm{a}}$ Patients may have had more than one febrile illness diagnosis during hospitalization. ${ }^{\mathrm{b}}$ Pneumonia, COPD exacerbation. ${ }^{\mathrm{c}} \mathrm{P}$. jirovecii pneumonia, disseminated M. avium, disseminated cytomegalovirus, Cryptococcus meningitis, cerebral or disseminated Toxoplasma. ${ }^{\mathrm{d}}$ Cellulitis, skin abscess. ${ }^{\mathrm{e}}$ Acute gastroenteritis, C. difficile colitis. 
Table 4. Descriptions of management outside standard guidelines.

\begin{tabular}{|c|c|c|c|}
\hline Management outside guidelines & Case group $(\mathrm{N}=35)$ & Comparator group $(\mathrm{N}=186)$ & $P$-value \\
\hline Total & $49 \%$ & $30 \%$ & 0.03 \\
\hline $\mathrm{PCP}^{\mathrm{a}}$ prophylaxis not indicated, given & $26 \%$ & $8 \%$ & 0.001 \\
\hline $\mathrm{PCP}^{\mathrm{a}}$ prophylaxis indicated, not given & $6 \%$ & $5 \%$ & 0.93 \\
\hline M. avium prophylaxis not indicated, given & $3 \%$ & $6 \%$ & 0.41 \\
\hline M. avium prophylaxis indicated, not given & $0 \%$ & $13 \%$ & 0.02 \\
\hline Multiple non-indicated laboratory tests ${ }^{\mathrm{b}}$ & $17 \%$ & $3 \%$ & 0.001 \\
\hline Non-indicated procedure ${ }^{c}$ & $11 \%$ & $3 \%$ & 0.02 \\
\hline Miscellaneous events & $3 \%$ & $1 \%$ & 0.18 \\
\hline
\end{tabular}

Patients that were managed outside standard guidelines based on absolute CD4 count had a median length of stay of 5 days versus 4 days compared to those that were managed per guidelines $(P=0.06)$. Case hospitalizations that were managed outside guidelines had a median length of stay of 7 days versus 3.5 days for those managed within guidelines in the comparator group $(P=$ 0.03 ) (Figure 2). There was no significant difference in the length of stay between cases and comparator group



Figure 2. Length of Stay Comparison. On the left hand side of chart is a comparison of appropriately managed hospitalizations to non-standard managed hospitalizations (regardless of whether they were cases or comparators) demonstrating a trend towards statistical significance $(P=0.06)$ of increased length of stay in those with non-standard management. On the right hand side of the chart is a comparison of appropriately managed hospitalizations in the comparator group (*Comp.) versus those who were managed in a non-standard fashion in the case group (cases) that was statistically different with a longer length of stay in the case group, $P=0.03$. patients $(P=0.52)$ who adhered to management guidelines or in those who did not adhere to management guidelines (cases versus comparator patients, $P=0.15$ ).

\section{Discussion}

We found that $49 \%$ of patients that presented with a marked decline in absolute CD4 count associated with preserved CD4 percentage during an acute illness were managed outside recommended DHHS and other relevant practice guidelines, compared with $30 \%$ of the comparator group. The case group also had prompt recovery of CD4 counts post admission and generally had lower pre-admission HIV-1 RNA levels. Deviation from management guidelines was minor in most circumstances and most commonly occurred due to administration of prophylaxis for $P$. jirovecii when it was probably not required. Adherence to guidelines was not affected by the involvement of a HIV specialist in the patient's care. Most importantly, length of stay was significantly prolonged in cases that were managed outside of recommended guidelines when compared to appropriately managed patients in the comparator group.

A trend towards extended length of stay was also seen in any hospitalization where management deviated from standard guidelines. Lastly, no persons in the case group with a low absolute CD4 count and preserved CD4 percentage were diagnosed with an AIDS related opportunistic infection.

Sudden declines in absolute CD4 count associated with acute illness in non HIV infected patients have been previously reported $[7,8]$. Beck investigated the effects of active pulmonary tuberculosis on lymphocyte subsets, finding that individuals infected with tuberculosis had significantly lower absolute CD4 counts than uninfected patients $\left(748\right.$ cells $/ \mathrm{mm}^{3}$ compared to 1043 cells $/ \mathrm{mm}^{3}$ ). 
No data regarding CD4 percentage was recorded by this group [7]. Williams evaluated the effects of various forms of acute infection on lymphocyte subpopulations. Patients with pneumonia or respiratory infections (574 cells $\left./ \mathrm{mm}^{3} ; 45 \%\right)$ and generalized sepsis $\left(669\right.$ cells $/ \mathrm{mm}^{3}$; 43.9\%) had significantly lower absolute CD4 counts and percentages than comparator groups of healthy patients aged $21-53$ years $\left(1075\right.$ cells $\left./ \mathrm{mm}^{3} ; 52.5 \%\right)$ and $67-88$ years $\left(924\right.$ cells $\left./ \mathrm{mm}^{3} ; 52.6 \%\right)$ [8]. Thus, it is not surprising that we found significant declines in the absolute CD4 count associated with acute illness in persons hospitalized with HIV infection.

The mechanism of the sudden decline in absolute CD4 count associated with acute illness or injury may be explained by a stress reaction that leads to a reduction in the total number of circulating lymphocytes and increase in the number of neutrophils, commonly known as a "left shift." As neutrophils demarginate and enter circulation, lymphocytes shift to the lymphatic-reticular systems, altering the balance within the bloodstream. There is no evidence that total body CD4 counts decline in this situation. Thus, the absolute CD4 count can appear falsely depressed. While the actual number of circulating lymphocytes has decreased, the relative CD4 percentage remains nearly stable during acute illness. This could also explain why the absolute CD4 count promptly returns to baseline once the stress has been removed. It is also reassuring that no patients with the relative decline in absolute CD4 count had an AIDS-related OI. This suggests that the CD4 population remains functional despite lower numbers in the bloodstream. It is unclear whether these sudden changes result in altered immune protection, as plasma CD4 cells represent $<2 \%$ of the total CD4 cells in the body.

Our results should be viewed carefully because of several important limitations. Our overall sample size may limit our ability to discern important differences. There were very few deaths, making it difficult to evaluate if management decisions would affect mortality. As in all retrospective studies, we did not have uniform data collection. Not all subjects were investigated equally, thus, AIDS defining OIs could have been missed. We believe this is unlikely since we reviewed subsequent hospitalizations and visits beyond the study period and no cases of AIDS defining OIs were found.

The management of acutely ill persons hospitalized with HIV is always challenging. It may be prudent to evaluate for AIDS-defining opportunistic infections in persons with measured CD4 counts $<200$ cells $/ \mathrm{mm}^{3}$ because one cannot be certain that patients are adhering to their antiretroviral therapy. Rapid declines in CD4 counts have been reported shortly after discontinuing antiretroviral therapy [9]. However, in these cases, typically the CD4 percentage declines as well. The inappropriate ad- ministration of prophylaxis for Pneumocystis jirovecii is unlikely to harm many patients. It is a very reasonable clinical decision to provide prophylaxis if $\mathrm{CD} 4$ criteria are met. However, the importance of this observation is that it was associated with prolonged length of stay which leads to added cost and the risk of harm. Indeed, the inappropriate use of antibiotics has been associated with emergence of resistant bacteria and should generally be avoided.

The phenomenon of the significant decline in CD4 count with preserved CD4 percentage appears to occur in HIV infected patients on stable antiretroviral therapy with acute febrile illnesses or severe stressful conditions. Discordant results between absolute inpatient and prior recent outpatient CD4 counts and percentages in persons on stable antiretroviral therapy should raise suspicion amongst clinicians. CD4 count risk stratification based upon the value obtained during an acute illness requiring hospitalization may not always be accurate. Administration of unnecessary antibiotics, overutilization of procedures, and excessive ordering of laboratory assays may result from inappropriate risk stratification. Providers tend to extend periods of inpatient observation in persons with depressed CD4 counts to ensure resolution of illness. These factors lead to prolonged hospitalizations with increased risk of complication to the patient and cost to the health care system. In conclusion, clinicians should review prior and current CD4 counts, HIV viral loads, and antiretroviral treatment history to adequately risk stratify patients with HIV infection presenting with acute illnesses to ensure that they avoid unnecessary interventions and prolongation of hospitalization.

\section{Acknowledgements}

Potential Conflicts of Interest: SMB: None. CJF: None. No financial support was received for the development and performance of this study. The authors thank Ms. Susan Groh for her expertise and assistance in the conduct of this project.

\section{REFERENCES}

[1] J. L. Fahey, J. M. G. Taylor, R. Detels, et al., "The Prognostic Value of Cellular and Serologic Markers in Infection with Human Immunodeficiency Virus Type 1," The New England Journal of Medicine, Vol. 322, No. 3, 1990, pp. 166-172. doi:10.1056/NEJM199001183220305

[2] J. P. Phair, A. Munoz, R. Detels, et al., "The Risk of Pneumocystis carinii Pneumonia among Men Infected with Human Immunodeficiency Virus Type 1," The New England Journal of Medicine, Vol. 322, No. 3, 1990, pp. 161-165. doi:10.1056/NEJM199001183220304

[3] J. E. Kaplan, H. Masur and K. K. Holmes, "Guidelines for Preventing Opportunistic Infections among HIV-Infected Persons: Recommendations of the US Public 
Health Service and the Infectious Diseases Society of America," MMWR-Recommendations and Reports, 51 (RR08), 14 June 2002, pp. 1-46.

[4] C. A. Benson, J. E. Kaplan, H. Masur, A. Pau and K. K. Holmes, "Treating Opportunistic Infections among HIVInfected Adults and Adolescents: Recommendations from CDC, the National Institutes of Health, and the HIV Medicine Association/Infectious Diseases Society of America," MMWR-Recommendations and Reports, 53 (RR15), 17 December 2004, pp. 1-112.

[5] J. A. Aberg, J. E. Gallant, J. Anderson, et al., "Primary Care Guidelines for the Management of Persons Infected with Human Immunodeficiency Virus: Recommendations of the HIV Medicine Association of the Infectious Diseases Society of America," Clinical Infectious Diseases, Vol. 39, No. 5, 2004, pp. 609-629. doi:10.1086/423390

[6] L. A. Mandell, R. G. Wunderink, A. Anzueto, et al., "Infectious Diseases Society of America/American Thoracic Society Consensus Guidelines on the Management of Community-Acquired Pneumonia in Adults," Clinical In- fectious Diseases, Vol. 44, Suppl. 2, 2007, pp. S27-S72. doi:10.1086/511159

[7] J. S. Beck, R. C. Potts, T. Kardjito and J. M. Grange, "T4 Lymphopenia in Patients with Active Pulmonary Tuberculosis," Clinical \& Experimental Immunology, Vol. 60, No. 1, 1985, pp. 49-54.

[8] R. C. Williams Jr., F. T. Koster and K. A. Kilpatrick, "Alterations in Lymphocyte Cell Surface Markers during Various Human Infections," The American Journal of Medicine, Vol. 75, No. 5, 1983, pp. 807-816. doi:10.1016/0002-9343(83)90412-6

[9] R. Seng, C. Goujard, L. Desquilbet, M. Sinet, C. Rouzioux, C. Deveau, F. Boufassa, J. F. Delfraissy, L. Meyer, A. Venet and The ANRS PRIMO and SEROCO Study Groups, "Rapid CD4+ Cell Decrease after Transient cART Initiated during Primary HIV Infection (ANRS PRIMO and SEROCO Cohorts)," Journal of Acquired Immune Deficiency Syndromes, Vol. 49, No. 3, 2008, pp. 251-258. doi:10.1097/QAI.0b013e318189a739 